Artículo de Revisión / Review Article

http://dx.doi.org/10.18004/mem.iics/1812-9528/2020.018.02.74

\title{
Bloqueo interauricular avanzado en pacientes con hipertensión arterial sistémica y fibrilación auricular
}

\author{
Rocío del Pilar Falcón Fleytas $^{1}$, Karina Elizabeth Scavenius Aguilera $^{1,2}$, Alfredo J. Meza , \\ Osmar Antonio Centurión ${ }^{1,2}$ \\ ${ }^{1}$ Universidad Nacional de Asunción, Hospital de Clínicas, División de Medicina Cardiovascular. \\ San Lorenzo, Paraguay \\ ${ }^{2}$ Sanatorio Metropolitano, Departamento de Investigación en Ciencias de la Salud. \\ Fernando de la Mora. Paraguay
}

Cómo referenciar este artículo/ How to reference this article:
Falcón Fleytas RP, Scavenius Aguilera KE, Meza AJ, Centurión OA. Bloqueo interauricular avanzado en pacientes con hipertensión arterial sistémica y fibrilación auricular. Mem. Inst. Investig. Cienc. Salud. 2020; 18(2): 74-85

\section{RE S U M E N}

La hipertensión arterial sistémica (HTA) continúa siendo un factor de riesgo de indudable importancia en el proceso del desarrollo de la enfermedad cardiovascular y la fibrilación auricular (FA). La FA constituye la arritmia sostenida más frecuentemente detectada. Es primordial dar el énfasis necesario a la prevención y al diagnóstico precoz de enfermedades con gran impacto social, médico y económico a la salud pública. En este contexto, es importante detectar la enfermedad en estadio subclínico e identificar factores que determinen con gran confiabilidad la aparición y desarrollo de una enfermedad. El electrocardiograma (ECG) es un método auxiliar de diagnóstico seguro, económico, de fácil manejo y accesible en prácticamente todos los centros médicos, y que nos sirve para detectar diversas entidades nosológicas. El bloqueo interauricular (BIA) diagnosticado por el ECG ocurre debido a un enlentecimiento de conducción entre las aurículas derecha e izquierda a causa de un retardo en la conducción de impulsos a través del haz de Bachmann. El sustrato anatómico para BIA está producido por la remodelación auricular debido a fibrosis, induciendo disincronía interauricular. El BIA está presente hasta en un 59\% de los pacientes mayores de la población general y estuvo directamente asociado a la HTA y la FA. Además, el BIA avanzado se asoció con un aumento del riesgo de unas 3 veces más de FA de nueva aparición y accidente cerebrovascular isquémico. Por lo tanto, es imprescindible realizar una investigación prospectiva adicional para determinar las estrategias óptimas en el adecuado manejo terapéutico de estos pacientes.

Palabras clave: bloqueo interauricular, fibrilación auricular, hipertensión arterial.

\section{Advanced interatrial block in patients with systemic arterial hypertension and atrial fibrillation}

\begin{abstract}
Systemic arterial hypertension (AHT) continues to be a risk factor of undoubted importance in the process of development of cardiovascular disease and atrial fibrillation (AF). AF is the most frequently detected sustained arrhythmia. For this reason, it is essential to give the necessary emphasis to the prevention and early diagnosis of diseases with great social, medical and economic impact on public health. In this context, it is important to detect the subclinical stage disease and identify factors that determine with great reliability the appearance and development of a
\end{abstract}

Fecha de recepción: junio 2020. Fecha de aceptación: julio 2020

* Autor correspondiente: Prof. Dr. Osmar Antonio Centurión, MD, PhD, FACC, FAHA. Professor of Medicine. Asuncion National University (UNA). Department of Health Sciences Investigation. Sanatorio Metropolitano. Teniente Ettiene 215 c/ Ruta Mariscal Estigarribia. Fernando de la Mora. Paraguay.

Email: osmarcenturion@hotmail.com 
disease. The electrocardiogram (ECG) is a safe, inexpensive auxiliary diagnostic method, easy to use and accessible in practically all medical centers, and which serves to detect various nosological entities. The interatrial block (IAB) diagnosed by the ECG occurs due to a slowing down of conduction between the right and left atria due to a delay in the conduction of impulses through the Bachmann pathway. The anatomical substrate for $I A B$ is produced by atrial remodeling due to fibrosis, inducing interatrial dyssynchrony. IAB is present in up to $59 \%$ of older patients in the general population and was directly associated with $\mathrm{AHT}$ and $\mathrm{AF}$. In addition, advanced IAB was associated with a 3-fold increased risk of new-onset AF and ischemic stroke. Therefore, it is essential to carry out additional prospective research to determine the optimal strategies in the adequate therapeutic management of these patients.

Keywords: interatrial block, atrial fibrillation, arterial hypertension.

\section{NTRODUCCIÓN}

En el desarrollo de enfermedades cardiovasculares intervienen numerosos factores, modificables y no modificables, entre ellos el de mayor prevalencia y gran importancia clínica es la hipertensión arterial sistémica $(H T A)^{(1-4)}$. Se estima que cerca del $40 \%$ de los hipertensos desconoce que lo es, el $20 \%$ de los diagnosticados no se trata con medicación antihipertensiva y cerca de la mitad de los casos diagnosticados, que reciben tratamiento no alcanza el objetivo terapéutico ${ }^{(5)}$. La hipertensión arterial continúa siendo un factor de riesgo de indudable importancia en el proceso del desarrollo de la enfermedad cardiovascular y la fibrilación auricular ${ }^{(6)}$.

La fibrilación auricular (FA) constituye la arritmia sostenida más frecuentemente detectada. Su prevalencia varía según la población en estudio, a nivel global, se estima que afecta a $1,5-2 \%$ de la población general ${ }^{(7-9)}$. Este porcentaje aumenta gradualmente si tenemos en cuenta sujetos ancianos, llegando incluso al $17 \%$ en los sujetos mayores de 80 años ${ }^{(10-14)}$. Sin embargo, aumenta aún más si tenemos en cuenta ciertas poblaciones especiales. Se observó que, en enfermos renales crónicos estudiados con Holter de $24 \mathrm{hs}$, la frecuencia de FA llega al $27 \%^{(10)}$. Esta patología está asociada, ya sea como causa o consecuencia, a varias patologías cardiovasculares: hipertensión arterial, diabetes mellitus, enfermedad renal crónica y en última instancia insuficiencia cardiaca ${ }^{(15-18)}$.

La medicina es la ciencia del perpetuo cambio. En este contexto, actualmente hay tendencia a hacer hincapié en la prevención y diagnóstico precoz de enfermedades con gran impacto en los pacientes y en el que generen grandes costos a la salud pública. Además de detectar la enfermedad en estadio subclínico o identificar factores que, al aparecer, determinen con gran confiabilidad la aparición de determinada condición ${ }^{(19-21)}$. Alexander $\mathrm{B}$, et al. ${ }^{(22)}$ observaron que determinadas variables de la onda P (morfología, voltaje, y duración) en el electrocardiograma (ECG) de 12 derivaciones permite identificar a los pacientes con riesgo de desarrollar FA, y estudiaron estos parámetros electrocardiográficos a fin de estratificar a los pacientes y realizar un seguimiento más estricto de aquellos con alta probabilidad de desarrollar FA.

Un parámetro detectado por el ECG es el bloqueo interauricular (BIA) que ocurre debido a un retraso de conducción entre las aurículas derecha e izquierda a causa de un retardo en la conducción de impulsos a través del haz de Bachmann. El BIA es un predictor significativo para el desarrollo de la fibrilación auricular (FA) ${ }^{(23-26)}$. El sustrato anatómico para el BIA está producido por la remodelación auricular debido a fibrosis, induciendo disincronía interauricular. La disfunción electromecánica de la aurícula izquierda (Al) desarrolla ciertos trastornos como activación anormal de la Al, aumento presión de la Al, dilatación de la Al, disfunción endotelial, y fibrosis auricular. Estas alteraciones fisiopatológicas favorecen el desarrollo de la FA con sus complicaciones tromboembólicas ${ }^{(27-30)}$. El BIA avanzado (Figura 1) ocurre secundariamente a la conducción auricular interrumpida o enlentecida, lo que puede conducir a cambios en períodos refractarios auriculares y la aparición de latidos auriculares prematuros, desencadenantes de arritmias auriculares ${ }^{(22-26)}$. EI BIA puede servir como un valioso marcador electrocardiográfico para evaluar el riesgo de nueva aparición de FA. Por lo tanto, es nuestra intención realizar un análisis del papel que posee el BIA en pacientes 
hipertensos y en la fibrilación auricular. Es fundamental poseer una mejor comprensión del BIA en ciertas subpoblaciones de pacientes para refinar los criterios de selección diagnósticos para candidatos que se beneficiarían de un monitoreo cardíaco ambulatorio extendido para detectar FA, además de estrategias informativas para el manejo adecuado de la anticoagulación.

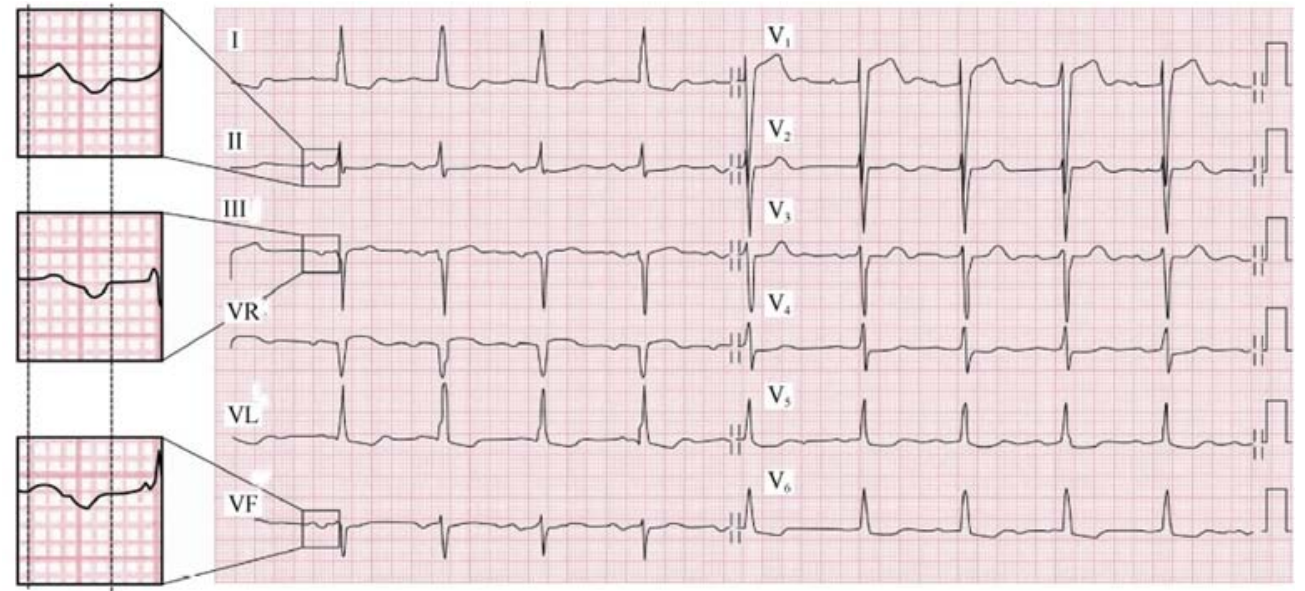

Figura 1: Bloqueo interauricular avanzado. Se observa una Onda P con una duración mayor de $200 \mathrm{~ms}$, con una morfología de patrón difásico (positivo-negativo) con negatividad terminal en las derivaciones electrocardiográficas inferiores II, III, y aVF. Reimpreso con permiso de Bayés de Luna A, Baranchuk A, Escobar Robledo LA, Massó van Roessel A, Martínez Sellés M. Diagnosis of interatrial block. J Geriatric Cardiol 2017; 14: 161-65.

\section{CONCEPTOS EPI DEMI OLOGI COS}

Las patologías del sistema circulatorio son la primera causa de muerte tanto a nivel mundial como en nuestro país, las dos principales causas englobadas en este conjunto de enfermedades son la cardiopatía isquémica coronaria y el accidente cerebrovascular, que en conjunto producen más del $50 \%$ de la mortalidad total ${ }^{(1,2)}$. Para el desarrollo de dichas patologías intervienen numerosos factores, modificables y no modificables, entre ellos el de mayor prevalencia y gran importancia clínica es la HTA. Se estima que cerca del $40 \%$ de los hipertensos desconoce que lo es, el $20 \%$ de los diagnosticados no se trata con medicación antihipertensiva y cerca de la mitad de los casos diagnosticados, que reciben tratamiento no alcanza el objetivo terapéutico ${ }^{(2-}$ 4).La HTA continúa siendo un factor de riesgo de indudable importancia en el proceso del desarrollo de la enfermedad cardiovascular y la fibrilación auricular ${ }^{(5)}$. Constituye el principal factor de riesgo modificable, asociado a las mayores causas de morbimortalidad cardiovascular a nivel mundial ${ }^{(6)}$.

En los últimos decenios los avances en el diagnóstico y en el tratamiento farmacológico de la fibrilación auricular y la hipertensión arterial sistémica y otros factores de riesgo cardiovascular como la hipercolesterolemia y la diabetes mellitus tipo 2 (DM2) han sido espectaculares y se traducen en un mayor grado de control ${ }^{(1-3)}$. No obstante, está aún lejos de los niveles óptimos deseables, debido en parte a una baja adherencia y a colectivos médicos con una elevada inercia terapéutica ${ }^{(2)}$.

En nuestro país se realizó el primer trabajo de investigación epidemiológica entre los años 1993-1995, en el que se detectó un 42\% de pacientes hipertensos; estas cifras se mantuvieron en los estudios posteriores ${ }^{(3)}$. La hipertensión arterial constituye un problema de salud pública dada su alta frecuencia y la carga en salud que representa, ya sea por su elevada morbilidad como por su letalidad. La prevalencia real de esta enfermedad varía enormemente, aumentando a mayor edad del sujeto. De esta manera, en los países desarrollados con poblaciones más añosas, más de dos tercios de los adultos padecen de hipertensión arterial sistémica ${ }^{(6)}$. 


\section{MECANISMOS FI SI OPATOLÓGI COS}

Ingresando al ámbito de la fisiopatología, la presión arterial corresponde a la tensión en la pared vascular que genera el flujo de la sangre en ella. Está determinada por el producto de dos factores: el gasto cardiaco y la resistencia periférica total. A su vez, el gasto cardiaco depende de la contractilidad miocárdica y del volumen circulante intratorácico ${ }^{(1)}$. En cambio, la resistencia periférica depende del tono de las paredes de la arteria y las características estructurales de la misma. Cada latido cardiaco sólo inyecta sangre al árbol arterial durante la fase sistólica ventricular, lo que determina un flujo pulsátil sobre las paredes arteriales ${ }^{(6)}$. El máximo valor de la presión durante la sístole se conoce como PA sistólica y el mínimo durante la diástole PA diastólica; la primera depende fundamentalmente del gasto cardiaco y de la distensibilidad de la aorta y otras arterias de gran calibre; en cambio la presión arterial diastólica depende de la resistencia periférica ${ }^{(5)}$.

La presión arterial es una variable biológica, por lo tanto, se trata de una variable continua. La elevación progresiva de la presión arterial condiciona numerosas alteraciones estructurales y funcionales en órganos diana, principalmente corazón, cerebro, ojos, riñones ${ }^{(1-3)}$. Esto desencadena las principales complicaciones relacionadas a la hipertensión arterial ya sea accidente cerebrovascular (ACV), infarto agudo de miocardio, enfermedad arterial periférica, fibrilación auricular, enfermedad renal crónica y todas las causas de mortalidad cardiovascular ${ }^{(6)}$.

Está ampliamente demostrado que la prevención, tanto primaria como secundaria, es la estrategia que más ha mejorado la calidad y cantidad de vida de los pacientes, basándose sobre todo en el control de los factores de riesgo cardiovascular (FRCV) modificables ${ }^{(2)}$. Entre ellos, el principal es la hipertensión arterial, además de las dislipidemias, hiperglicemia, y alteraciones del peso corporal. Al respecto, se ha demostrado que el control de los FRCV fue mejor ante la presencia de enfermedad coronaria, en pacientes de mayor edad y con menos de $3 \mathrm{FRCV}^{(4)}$. Como delineamiento general, en todos ellos se enfatizó el pobre control de los factores de riesgo y la necesidad de intensificar el control de los mismos, para que, de esta manera la mayor parte de los pacientes presente valores en el rango adecuado según la estratificación de riesgo individual ${ }^{(4-6)}$.

Una de las complicaciones más significativas de la HTA, tanto por la morbilidad debido a la patología en sí como por los posibles eventos clínicos es la fibrilación auricular. La HTA predispone a la aparición de diversas arritmias, debido a las alteraciones de la estructura y la función del ventrículo izquierdo de larga duración. Esto da lugar a aumento de la presión en la aurícula izquierda, dilatación de la cavidad y deterioro de la función de la misma. Este remodelado estructural y funcional auricular puede aumentar el riesgo de producción de arritmias auriculares. Las arritmias potencialmente más graves son las ventriculares pero las más frecuentes son las supraventriculares siendo la más frecuente la fibrilación auricular ${ }^{(4-6)}$. El remodelado auricular estructural puede ocasionar cambios fibróticos en el miocardio auricular que pueden generar trastornos electrofisiológicos. Estos cambios producen retardo en la conducción, bloqueos direccionales en el miocardio auricular que generan electrogramas auriculares anormales, fraccionados y prolongados que se pueden registrar mediante un mapeo por catéter endocárdico auricular en ritmo sinusal (Figura 2). 

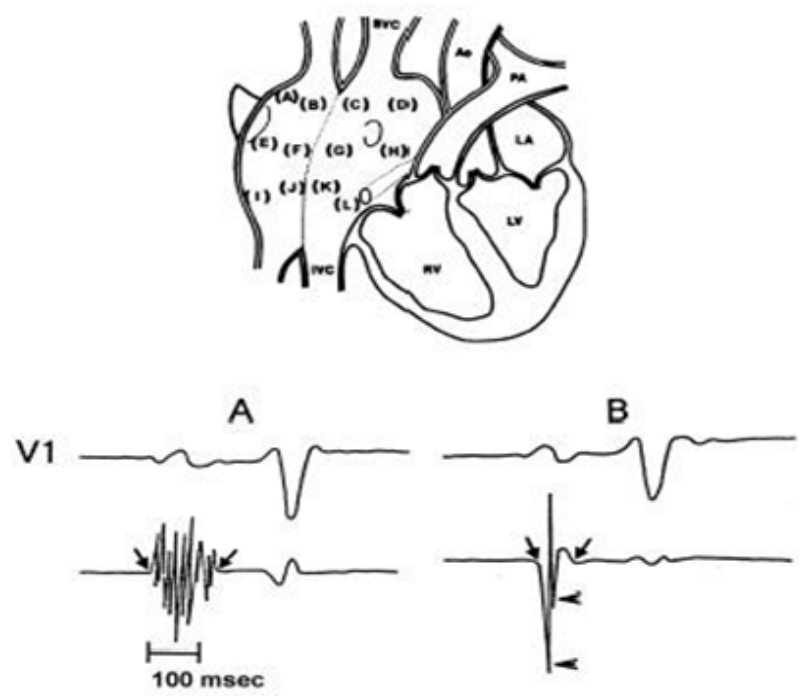

Figura 2: Sitios de mapeo endocárdico por catéter de la aurícula derecha. Los electrogramas auriculares fueron registrados de 12 sitios endocárdicos en cada paciente. Los sitios que figuran de la $\mathrm{A}$ a la $\mathrm{D}$ corresponden a la parte alta de la aurícula derecha mientras que los sitios de la $\mathrm{E}$ a la $\mathrm{H}$ son de la parte media, y aquellos de la I a la $\mathrm{L}$ son de la parte baja. Los sitios marcados como A, E y I corresponden a la región anterior, los sitios B, F y J a la región lateral, los sitios $C, G$ y $K$ a la región posterior, y los sitios $\mathrm{D}, \mathrm{H}$ y $\mathrm{L}$ corresponden a la región medial de la aurícula derecha. SVC: vena cava superior. IVC: vena cava inferior. Ao: Aorta. PA: arteria pulmonar. LA: aurícula izquierda. RV: ventrículo derecho. LV: ventrículo izquierdo. En la parte inferior $(A)$ se observa un electrograma auricular endocárdico anormal con una duración de 130 ms y 10 deflecciones fragmentadas. (B) demuestra un electrograma auricular endocárdico normal que tiene una duración de $80 \mathrm{~ms}$ y 2 deflecciones fragmentadas. Reimpreso con permiso de Centurion et al. Influence of advancing age on fractionated right atrial endocardial electrograms. Am J Cardiol 2005; 96:239-42.

\section{BLOQUEO I NTERAURI CULAR EN LA FI BRI LACIÓN AURI CULAR}

La fibrilación auricular es la arritmia crónica más frecuentemente detectada, su aparición condiciona un incremento de la morbimortalidad cardiovascular, además de tener un alto impacto en la calidad de vida de los pacientes. La prevalencia de esta enfermedad aumenta con la edad ${ }^{(13)}$. Se calcula que $0,95-1 \%$ de la población adulta tendrá en algún momento de su vida una FA. En el ámbito ambulatorio esta prevalencia aumenta hasta el $6 \%$ y puede llegar al $4 \%$ en los servicios de urgencias; se espera un aumento exponencial de la detección de FA por la mayor expectativa de vida actual de las personas, principalmente en países desarrollados ${ }^{(14)}$. Su tratamiento implica a varios grupos farmacológicos que se combinan para lograr diferentes objetivos individualizados a cada paciente ${ }^{(15)}$.

El tratamiento de esta patología resulta complejo; dependiendo de cada caso se puede optar por el control del ritmo o por el control de la frecuencia. Si bien, el control del ritmo no aumenta la sobrevida a largo plazo, permite aliviar la sintomatología y mejorar la hemodinamia. También se puede optar por simplemente buscar el control de la frecuencia cardiaca ${ }^{(16)}$. Si el mejor tratamiento para la FA es el control del ritmo o el control de la frecuencia es un tema debatido de mucha controversia hasta la actualidad $^{(17)}$.El tratamiento que sí demostró mejoría de la sobrevida fue la prevención del ACV. De hecho, uno de cada cinco ictus se atribuye a FA, lo cual aumenta al doble la tasa de mortalidad y la tasa de un nuevo ACV en cinco veces ${ }^{(8)}$. Este tratamiento consiste en la anticoagulación, para ello la escala $\mathrm{CHA}_{2} \mathrm{DS}_{2}-\mathrm{VASc}$, ha sido ampliamente validada para decidir la anticoagulación en la población general y de esta manera prevenir la aparición de eventos tromboembólicos ${ }^{(9-11)}$. Existe otra escala que valora el riesgo de sangrado: HASBLED. El balance entre ambas escalas determina en gran medida la seguridad del tratamiento anticoagulante, al no ser una medicación inocua, 
es de vital importancia estandarizar la indicación de la misma basándose en criterios validados en estudios previos con alto nivel de confiabilidad ${ }^{(15)}$.

Algunas condiciones asociadas suelen aumentar aún más la posibilidad de desarrollar esta arritmia. Entre ella se encuentra la cirugía cardiaca y algunas valvulopatías ${ }^{(17)}$. La FA corresponde a la arritmia más frecuente en la cirugía cardiaca, se observa en un 15-45\% de las intervenciones, independiente de la complejidad de la misma. Su aparición se asocia con estancias hospitalarias más largas, aumento de la morbilidad y mortalidad a los 6 meses ${ }^{(15-17)}$. La patogenia de la FA posoperatoria no se conoce por completo, pero se piensa que está relacionada con una combinación de factores perioperatorios, como la inflamación del pericardio, el aumento del tono simpático y la sobrecarga de volumen y un sustrato anatómico y funcional vulnerable en la aurícula izquierda ${ }^{(15-17)}$. En los pacientes con una estenosis aórtica grave, las alteraciones de la estructura y la función del ventrículo izquierdo de larga duración dan lugar a aumento de la presión en la aurícula izquierda, dilatación de la cavidad y deterioro de la función de la misma. Este remodelado estructural y funcional auricular puede aumentar el riesgo de arritmias auriculares, tanto antes como después de la intervención quirúrgica. Todo esto representa mayor riesgo de mortalidad para el paciente, por lo tanto, es sumamente importante la detección precoz de estos episodios e incluso detectar aquellos pacientes con mayor riesgo a desarrollarla para poder darle seguimiento más cercano, ya sea en una situación preoperatoria o después de ella ${ }^{(18)}$.

El bloqueo interauricular avanzado es una alteración que se detecta en el electrocardiograma (ECG) y está caracterizado por una duración de onda $P \geq 120 \mathrm{~ms}$ y una morfología bimodal de onda $\mathrm{P}$ (positivo/negativo) en las derivaciones inferiores (II, III y aVF) (19-21). La anormalidad eléctrica generalmente involucra el haz de Bachmann y se ha asociado con taquiarritmias auriculares, dilatación auricular izquierda, disfunción electromecánica auricular izquierda y tromboembolismo sistémico que incluye accidente cerebrovascular (22-24). La asociación entre el BIA avanzado y las arritmias auriculares, en particular la FA, es conocida como "Síndrome de Bayes" $(25,26)$.

Varios estudios realizados sobre el BIA han reportado una alta prevalencia de este bloqueo interauricular en entornos hospitalarios y ambulatorios, lo que llevó a algunos autores a referirse a este hallazgo electrocardiográfico como una pandemia infradiagnosticada ${ }^{(27-31)}$. De hecho, en un estudio que utilizó 1000 ECG no seleccionados, consecutivos, en pacientes hospitalizados, observó la presencia del BIA en el $47 \%$ de los pacientes que tenían ritmo sinusal normal ${ }^{(28)}$. En el ámbito ambulatorio, un estudio realizado en la población general de una comunidad de ancianos mayores de 65 años reveló que la prevalencia de BIA fue del 59\%,con una correlación significativa entre con la hipertensión arterial sistémica ${ }^{(29)}$. En otro estudio realizado sobre una población de la comunidad de ancianos de un programa de atención integral para personas mayores se encontró una prevalencia de BIA de $48,6 \%{ }^{(30)}$. Es evidente que el $\mathrm{BIA}$ es un hallazgo electrocardiográfico bastante frecuente, por ende, es importante buscarlo e identificarlo sobre todo en personas mayores.

Se considera que el BIA es un fuerte predictor del desarrollo de FA. Los pacientes con BIA poseen una desincronización interauricular que puede predisponerlos a desarrollar $\mathrm{FA}^{(31-34)}$. En un estudio de cohorte prospectivo con un seguimiento medio de 16 meses, la prevalencia de BIA fue comparado entre 308 pacientes que desarrollaron FA con un grupo control de 308 pacientes que permanecieron en ritmo sinusal durante el período de seguimiento. La prevalencia de BIA fue del $52 \%$ en la cohorte que desarrolló FA, en comparación con solo un $18 \%$ en la cohorte control ${ }^{(32)}$. En el estudio de riesgo de aterosclerosis en la comunidad (ARIC), que es un gran estudio prospectivo basado en la población, se demostró que los pacientes con BIA avanzado tenían tasas de FA significativamente más altas que aquellos sin BIA avanzado. Esta mayor incidencia de FA fue muy significativa incluso después del ajuste para factores demográficos, factores de riesgo cardiovascular, enfermedad de las arterias coronarias, e insuficiencia cardíaca congestiva ${ }^{(33)}$. En una 
pequeña cohorte de 97 pacientes, Sadiq et al $^{(27)}$, examinaron si el BIA avanzado podría predecir la FA de inicio reciente en pacientes con insuficiencia cardíaca grave que requieren terapia de resincronización cardíaca. Durante el seguimiento, se detectó FA en 29 pacientes y la incidencia fue mayor en pacientes con BIA avanzado comparados a aquellos pacientes $\sin \mathrm{BI}^{(27)}$.

En un metaanálisis anterior $\mathrm{T}$ se $\mathrm{G}$, et al. encontraron que la presencia del BIA predijo un nuevo inicio de FA, y en el análisis de subgrupos se alcanzó una significación estadística para el BIA avanzado, pero no para el BIA parcial ${ }^{(35)}$. EI HR agrupado fue de 2.58 (1.35-4.96). Por otro lado, He J, et al. observaron que las anormalidades de la onda $\mathrm{P}$ (onda $\mathrm{P}$ fuerza terminal en la derivación V1, duración de la onda $\mathrm{P}$ y área máxima de la onda $\mathrm{P}$ ), también predijo accidente cerebrovascular independientemente de $\mathrm{FA}^{(36)}$. Dado su asociación con la dilatación auricular izquierda y con la disfunción auricular electromecánica, el BIA puede facilitar el sustrato anatómico-eléctrico para la formación de trombos intraauriculares y la embolia posterior. Lorbar $\mathrm{M}$, et al. estudiaron en forma retrospectiva a 104 pacientes con accidentes cerebrovasculares embólicos y observaron que el BIA estaba presente en un $80 \%$ de estos pacientes que estaban en ritmo sinusal (24). Otro análisis retrospectivo, Ariyarajah $\mathrm{V}$, et $\mathrm{al}^{(37)}$. compararon 85 pacientes con accidente cerebrovascular embólico con 208 pacientes con accidente cerebrovascular no embólico, que fueron emparejados por factores de riesgo de accidente cerebrovascular, revelaron que $\sim 88 \%$ estaban en ritmo sinusal, pero la prevalencia de BIA fue significativamente mayor en la cohorte de accidente cerebrovascular embólico $(60.6 \%)$ en comparación con la cohorte de accidente cerebrovascular no embólico (39.8\%), y esta diferencia fue estadísticamente significativa ${ }^{(37)}$. Los factores de riesgo para el BIA avanzado, FA y accidente cerebrovascular posterior parecen ser muy similares, y la patogénesis subyacente probablemente se deba a fibrosis miocárdica y remodelación auricular ${ }^{(37-43)}$. Estos cambios de remodelación tanto a nivel estructural como a nivel electrofisiológico producen electrogramas auriculares anormales Un electrograma endocárdico auricular anormalmente prolongado y fraccionado registrado en estos pacientes mediante el mapeo de la aurícula derecha durante ritmo sinusal, podría traducir una actividad eléctrica localizada y no homogénea relacionada con una conducción retardada, no uniforme y anisotrópica a través de un miocardio auricular patológico ${ }^{(44-47)}$ (Figura 3 ). Además, se ha demostrado histológicamente que los tejidos donde se originan los electrogramas anormalmente prolongados y fraccionados presentan procesos fibro-degenerativos ${ }^{(48-52)}$. Las causas probables de interrupción del haz de Bachmann incluyen isquemia, enfermedad degenerativa del envejecimiento, enfermedades infiltrativas, enfermedad coronaria difusa y afecciones inflamatorias. EI BIA avanzado ocurre secundario a la conducción auricular interrumpida, lo que puede conducir a cambios en períodos refractarios auriculares y la aparición de latidos auriculares prematuros, desencadenantes de arritmias auriculares ${ }^{(22-26)}$. EI BIA puede servir como un valioso marcador electrocardiográfico para evaluar el riesgo de nueva aparición de FA. 

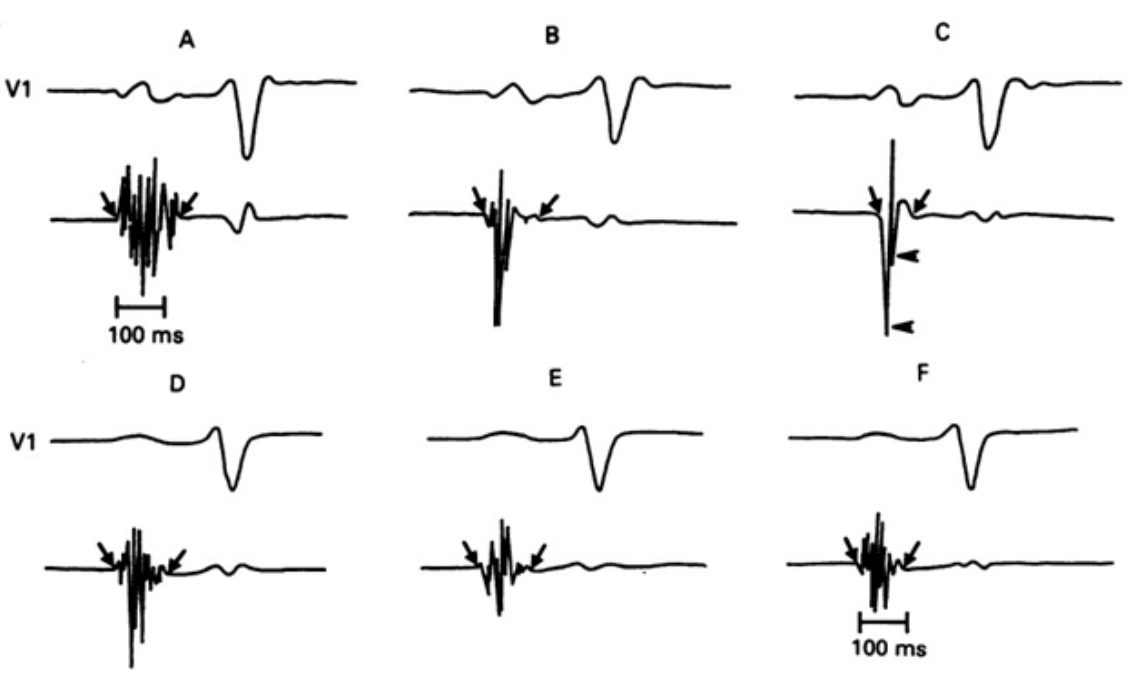

Figura 3. Seis electrogramas auriculares endocárdicos registrados en diferentes sitios de la aurícula derecha y registrados por medio de un mapeo por catéter endocárdico de la aurícula derecha en ritmo sinusal en dos pacientes con disfunción del nodo sinusal (DNS) ( $A, B$ y C en un paciente con DNS con Rubenstein Tipo I y II; D, E y F en un paciente con DNS con Rubenstein Tipo III). Las flechas diagonales representan el inicio y el desplazamiento de la actividad eléctrica local y las flechas horizontales las desviaciones hacia debajo de la isoeléctrica formando las deflecciones del electrograma. Reimpreso con permiso de Centurión OA, Fukatani M, Konoe A, Tanigawa M, Shimizu A, Isomoto S y col. Different distribution of abnormal endocardial electrogram with in the right atrium in patients with sick sinuss yndrome. $\mathrm{Br}$ Heart J 1992; 68: 596-600.

En conclusión, el BIA es una entidad poco reconocida en la práctica clínica habitual. En el mundo real, el IAB está presente en el $47 \%$ y el $59 \%$ de los pacientes mayores de la población general y estuvo directamente asociado a la hipertensión arterial sistémica y la fibrilación auricular. Además, el BIA avanzado se asoció con un aumento del riesgo de unas 3 veces más de FA de nueva aparición y accidente cerebrovascular isquémico. Debido a la relativamente alta prevalencia del BIA y sus riesgos significativos asociados, es imprescindible realizar una investigación prospectiva adicional para determinar las estrategias óptimas en el adecuado manejo terapéutico de estos pacientes.

\section{REFERENCI AS BIBLI OGRÁFI CAS}

1. Brotons Cuixart C, Alemán Sánchez JJ, Banegas Banegas JR, Fondón León C, Lobos-Bejarano JM, Martín Rioboó E, et al. Recomendaciones preventivas cardiovasculares. Actualización PAPPS 2018. Aten Primaria. 2018;50:4-28. doi: 10.1016/S0212-6567(18)30360-3.

2. Báez L, Blanco $M$, Bohórquez $R$, Botero R, Cuenca G. Definición-Diagnostico de la hipertensión arterial; Aspectos epidemiológicos. Rev Colomb Cardiol. 2007; 13(sup 1): 195-6.

3. Ortellado J, Ramírez A, González G, Olmedo G, Ayala M, Sano M, et al. Consenso paraguayo de Hipertension Arterial 2015. Rev virtual Soc Parag Med Int. 2016;3(2): 11-57.

4. Tagle R. Diagnóstico de hipertensión arterial. Rev med Clín Condes. 2018;29(1): 12-20.

10.1016/j.rmclc.2017.12.005
5. Tamayo-Ojeda C, Parellada-Esquius N, Salvador-González B, Oriol-Torón PÁ, Rodríguez-Garrido MD, et al. Seguimiento de las recomendaciones en prevención secundaria cerebrovascular en atención primaria. Atención Primaria. 2017;49(6): 351-8.

doi: 10.1016/j.aprim.2016.07.004

6. Williams B, Mancia G, Spiering W, Rosei EA, Azizi M, Burnier M, et al. 2018 ESC/ESH Guidelines for the management of arterial hypertension. European Heart J ournal. 2018;39:30214.https://doi.org/10.1093/eurheartj/eh y339

7. Zanetti FL. La fibrilación auricular y los anticoagulantes directos. Rev Med Chil. 2016; 144(9): 1101-2.

http://dx.doi.org/10.4067/S003498872016000900001

8. Giner-Soriano M, Casajuana M, RosoLlorach A, Vedia C, Morros R. 
Effectiveness, safety and costs of stroke prevention in non-valvular auricular fibrillation. Study of cohorts matched by Propensity score. Aten Primaria. 2020; 52(3): 176-84.doi:

10.1016/j.aprim.2019.06.002

9. Belmar Vega L, de Francisco ALM, Bada da Silva J, Galván Espinoza L, Fernández Fresnedo G. Nuevos anticoagulantes orales en pacientes con enfermedad renal crónica. Nefrología. 2017;37(3): 244-

52.http://dx.doi.org/10.1016/j. nefro. 20 16.08 .006

10. Sánchez Soriano RM, Albero Molina MD, Chamorro Fernández $\mathrm{Cl}$, Juliá-Sanchís $R$, López Menchero R, Del Pozo Fernández $\mathrm{C}$, et al. Impacto pronóstico a largo plazo de la anticoagulación en pacientes en hemodiálisis con fibrilación auricular. Nefrologia. 2018; 38(4):394400. DOI: $10.1016 /$ j. nefro.2017.11.026

11. Fernandez de Simón AF, Coll-Vinent $B$, Martín A, Suero C, Sánchez J, Varona $M$, Sanchez S, Varona $S$ et al. Cardioversion in recent onset atrial fibrillation. Emergencias. 2019; 31(4): 227-33.

12. White JL, Hollander JE. Fibrilación auricular rápida, cardioversión rápida, regreso a casa rápido. Emergencias. 2019; 31:223-4.

13. Sandoya $D E$, Aguilar $P$, Vázquez $H$. Prevalencia de la fibrilación auricular en la población adulta de Montevideo. Rev Uruguaya Cardiol. 2014; 29(2): 187-91.

14. Jacob J, Cabello I, Yuguero O, Alexis Guzmán J, Arranz Betegón M, Abadías $M J$, et al. Registro de fibrilación auricular en servicios de urgencias del Institut Català de la Salut (URGFAICS): análisis en función del tipo de fibrilación auricular y de la reconsulta a urgencias relacionada a los 30 días. Emergencias. Rev la Soc Española Med Emergencias. 2019; 31(2): 99-106.

15. del Arco Galán C. Medicamentos, fibrilación auricular permanente y problemas derivados. Emergencias. 2016;28(2): 71-2.

16. De Simón AF, Coll-Vinent $B$, Martín A, Suero C, Sánchez J, Varona $M$, et al. Cardioversion in recent onset atrial fibrillation. 2019; 31(4): 227-33.

17. White JL, Hollander JE. Fibrilación auricular rápida, cardioversión rápida, regreso a casa rápido. Emergencias. 2019; 31:223-4.

18. Pessoa-Amorim G, Mancio J, Vouga L, Ribeiro J, Gama V, Bettencourt N, et al. Alteración del strain auricular izquierdo como predictor de fibrilación auricular de nuevo comienzo tras recambio valvular aórtico, independientemente del tamaño de la aurícula izquierda. Rev Esp Cardiol. 2017;71(6):466-76.DOI: 10.1016/j.recesp. 2017.09.020

19. Bayés de Luna $A$, Cladellas $M$, Oter $R$, Torner P, Guindo J, Martí V, et al. Interatrialconduction block and retrograde activation of the left atrium and paroxismal supraventricular tachy arrhythmia. Eur Heart J 1988;9:11128.DOI:

10.1093/oxfordjournals. eurheartj.a0624 07

20. Bayes de Luna A, Fort de Ribot R, Trilla E, Julia J, Garcia J, Sadurni J, et al. Electrocardiographic and vector cardiographic study of interatrial conduction disturbances with left atrial retrograde activation. J Electrocardiol 1985; 18: 1-

13. https: // doi.org/10.1016/S00220736(85)80029-7

21. Bayés de Luna A, Oter MC, Guindo J. Interatrial conduction block with retrograde activation of the left atrium and paroxysmal stupra ventricular tachy arrhythmias: influence of preventive antiarrhythmic treatmen. Int J Cardiol 1989; 22:147-50.doi: 10.1016/01675273(89)90061-2.

22. Alexander B, Milden J, Hazim B, Haseeb $S$, Bayes-Genis A, Elosua R, et al. New electrocardiographic score for the prediction of atrial fibrillation: The MVP ECG risk score (morphology-voltage-Pwave duration). Ann Noninvasive Electrocardiol. $2019 ; 24(6)$ : 1-7.doi: 10.1111/anec. 12669.

23. Goyal SB, Spodick DH. Electromechanical dysfunction of the left atrium associated with interatrial block. Am Heart J 2001;142:82327.DOI: 10.1067/mhj.2001.118110

24. Lorbar $M$, Levrault $R$, Phadke JG, Spodick DH. Interatrial block as a predictor of embolicstroke. Am J Cardiol 2005; 95:667-68.DOI: 10.1016/j.amjcard.2004.10.059

25. Conde D, Baranchuk A. Interatrial block as anatomical-electrical substrate for supra ventricular arrhythmias: Bayes' Syndrome. Arch Mex Cardiol 2014; 84: 32-40.DOI:

10.1016/j.acmx.2013.10.004

26. Bacharova L, Wagner GS. The time for naming the interatrial block syndrome: Bayes' Syndrome. J Electrocardiol 2015; 48: 133-34.doi:

10.1016/j.jelectrocard.2014.12.022.

27. SadiqAli F, Enriquez A, Conde D, Redfearn D, Michael K, Simpson C, et al. Advanced Interatrial Block Predicts New Onset Atrial Fibrillation in Patients with Severe Heart Failure and Cardiac 
Resynchronization

Noninvasive

Therapy. Ann Electrocardiol

2015; 20: 586-91.doi:

10.1111/anec. 12258.

28. Asad N, Spodick DH. Prevalence of interatrial block in a general hospital population. AmJ Cardiol 2003; 2003; 91:609-10. DOI : 10.1016/s0002-9149(02)03320-9

29, Ninios I, Pliakos C, Ninios V, Karvounis $H$, Louridas G. Prevalence of interatrial block ina general population of elderly people. Ann Noninvasive Electrocardiol 2007; 12: 298-300.

30. Ariyarajah V, Puri $P$, Kranis M, Wilner DA, Spodick DH. Prevalence of interatrial blockin the Program of AllInclusive Care for the Elderly (PACE). Am J GeriatrCardiol2006; 15: 174-7.DOI: 10.1111/j.1076-7460.2006.04518.x

31. Bernal E, Bayés-Genís A, Ariza-Solé A, Formiga F, Vidán MT, Escobar-Robledo LA, et al. Interatrial block, frailty and prognosis in elderly patients with myocardial infarction.

Electrocardiol2018; 51:1-7.doi:

10.1016/j.jelectrocard.2017.08.026.

32. Agarwal YK, Aronow WS, Levy JA, Spodick $\mathrm{DH}$. Association of interatrial block with development of atrial fibrillation. Am J Cardiol 2003; 91:882.doi: $\quad$ 10.1016/s00029149(03)00027-4.

33. O'Neal WT, Zhang ZM, Loehr LR, Chen LY, Alonso A, Soliman EZ. Electrocardiographic Advanced Interatrial Block and Atrial Fibrillation Risk in the General Population. Am J Cardiol 2016; 117: 1755-9.

34. Kristensen SL, J hund PS, Mogensen UM, Rørth R, Abraham WT, Desai A, et al. PARADIGM-HF and ATMOSPHERE Committees and Investigators. Prognostic Value of $\mathrm{N}$-Terminal Pro-BType Natriuretic Pepti de Levels in Heart Failure Patients With and Without Atrial Fibrillation. Circ Heart Fail 2017; 10.doi: 10.1161/CIRCHEARTFAI LURE.117.0044 09.

35. Tse G, Wong CW, Gong M, Wong WT, Bazoukis $\mathrm{G}$, Wong $\mathrm{SH}$, et al. International Health Informatics Study (IHIS) Network. Predictive value of inter-atrial block for new on set or recurrent atrial fibrillation: A systematic review and meta-analysis. Int J Cardiol 2018; 250: 152-56. doi: 10.1016/j.ijcard.2017.09.176.

36. He J, Tse G, Korantzopoulos P, Letsas $\mathrm{KP}$, Ali-Hasan-Al-Saegh S, Kamel H, etal. P-Wave Indices and Risk of Ischemic Stroke: A Systematic Review andMeta-Analysis.
2017; 48:2066-72.doi: 10.1161/STROKEAHA. 117.017293.

37. Ariyarajah V, Puri P, Apiyasawat S, Spodick DH. Interatrial block: a novel risk factor for embolicstroke? Ann Noninvasive Electrocardiol 2007; 12:1520.doi: $10.1111 /$ j.1542-

474X.2007.00133.X

38. Andersson C, Preis SR, Beiser A, DeCarli C, Wollert KC, Wang TJ, et al. Associations of Circulating Growth Differentiation Factor-15 and ST2ConcentrationsWithSubclinical

Vascular Brain Injury and Incident Stroke. Stroke2015;46:2568-75.doi: 10.1161/STROKEAHA.115.009026

39. Alexander B, MacHaalany J, Lam B, van Rooy H, Haseeb S, Kuchtaruk A, et al. Comparison of the Extent of Coronary Artery Disease in Patients With Versus Without Interatrial Block and Implications for New-Onset Atrial Fibrillation. Am J Cardiol 2017; 119: 1162-65. DOI: 10.1016/j.amjcard.2016.12.032

40. Hart RG, Pearce LA, Aguilar MI. Metaanalysis: antithrombotic therapy to prevent stroke in patients who haven on valvular atrial fibrillation. Ann Intern Med 2007; 146:857-67.doi: 10.7326/0003-4819-146-12200706190-00007.

41. Bayes de Luna A, Baranchuk A, Martínez-Sellés $M$, Platonov PG. Anticoagulation in patients at high risk of stroke without documented atrial fibrillation. Time for a paradigmshift? Ann Noninv Electrocardiol 2017; 22: doi: 10.1111/anec.12417.

42. Poppe KK, Squire IB, Whalley GA, Køber L, McAlister FA, McMurray JJ, et al. Known and missing left ventricular ejection fraction and survival in patients with heart failure: a MAGGIC metaanalysis report. Eur J Heart Fail 2013; 15: 1220-7.doi:

10.1093/eurjhf/hft101.

43. Bayés de Luna $A$, Baranchuk $A$, Escobar Robledo LA, Massó van Roessel A, Martínez Sellés $M$. Diagnosis of interatrial block. J Geriatric Cardiol 2017; 14: 161-

65.doi: $10.11909 / \mathrm{j}$.issn.1671-

5411.2017.03.007

44. Centurión OA, Fukatani $M$, Shimizu $A$, Konoe $A$, I somoto S, Tanigawa $M$ et al. Anterograde and retrograde decremental conduction overleft-sided accessory atrioventricular path ways in the Wolff-Parkinson-White syndrome. Am Heart J 1993; 125: 1038-47.

45. Centurión OA, Fukatani $M$, Konoe $A$, Tanigawa $M$, Shimizu $A$, Isomoto $S$ et al. Different distribution of abnormal 
endocardial electrogram with in the right atrium in patients with sick sinus syndrome. Br Heart J 1992; 68:596600.

46. Centurión OA, Isomoto S, Fukatani M, Shimizu A, Konoe A, Tanigawa $M$, et al. Relationship between atrial conduction defects and fractionated atrial endocardial electrograms in patients with sick sinus syndrome. PACE 1993; 16: 2022-33.

47. Centurión OA, Fukatani $M$, Konoe $A$, Tanigawa M, Shimizu A, Isomoto $S$, et al. Electrophysiological abnormalities of the atrial muscle in patients with sinus node Dysfunction without tachy arrhythmias. Int J Cardiol 1992;37:4150.

48. Centurión OA, Shimizu A, Isomoto $S$, Konoe A, Kaibara $M$, Hayano $M$, et al. Influence of advancing age on fractionated right atrial endocardial electrograms. Am J Cardiol
2005; 96:239-42.doi:

10.1016/j.amjcard.2005.03.052.

49. Centurión OA, Isomoto $S$, Shimizu A, Konoe $A$, Yano $K$. The effects of agingonatrial endocardial electrograms in patients with paroxysmal atrial fibrillation. Clin Cardiol 2003;26:435-8. doi: $10.1002 / \mathrm{clc} .4960260911$

50. Shimizu A, Centurión OA. Electrophysiological properties of the human atrial fibrillation. Cardiovasc Res 2002; 54:302-14. doi: 10.4022/jafib. 232

51. Centurión OA, Isomoto S, Shimizu A. Electrophysiological changes of the atrium in patients with lone paroxysmal trial fibrillation. JAFIB 2010;1(12):65662.

52. Centurión OA. Age-related electrophysiological changes of the atrial myocardium in patients with paroxysmal atrial fibrillation. J Cardiol Cardiol Curr Res 2015;3(6):00121. DOI: $10.15406 /$ jccr.2015.03.00121 